BBA 76758

\title{
KINETICS OF THE VALINOMYCIN-INDUCED POTASSIUM ION LEAK FROM LIPOSOMES WITH POTASSIUM THIOCYANATE ENCLOSED
}

\author{
M. C. BLOK, J. DE GIER and L. L. M. VAN DEENEN \\ Laboratory of Biochemistrl, Unitersity of Utrecht, Transitoriam 3, Unitersity Centre "De Uithof", \\ Padualaan 8, Utrecht (The Netherlands)
}

(Received May 8th, 1974)

\section{SUMMARY}

The kinetics of the valinomycin-induced $\mathrm{K}^{+}$leak from egg lecithin liposomes with KCNS enclosed were studied by measuring the initial increase of the leak foilowing the addition of the ionophore. Variation of liposome and valinomycin concentrations enabled a discrimination between the affinity of the ionophore for the lipid bilayer and the turnover rate of the transport process. The results indicate that valinomycin is distributed immediately between the outer liposomal bilayer and the aqueous phase. A partition constant of $0.0222 \mathrm{~cm}$ at $30^{\circ} \mathrm{C}$ was obtained for egg lecithin liposomes. By varying the temperature it was shown that the partition constant has a negative temperature coefficient. The Arrhenius activation energy of the transport process itself is $15.7 \div 1.1 \mathrm{kcals} /$ mole. Concerning the mechanism of the valinomycinmediated transport it was found that the complex formation can be described kinetically by the formation of a ternary complex in which $\mathrm{K}^{+}$and a thiocyanate ion are bound by the ionophore.

By varying the fatty acid composition of the membrane constituents it was found that the turnover rate of the transport increased with increasing unsaturation of the phospholipids present in the membrane. This result suggests that the translocation of the complex is the rate-limiting step of the transport cycle. At $30^{\circ} \mathrm{C}$ a turnover constant of $328+44$ potassium ions/molecule valinomycin per second was obtained for egg lecithin lipsomes.

\section{INTRODUCTION}

Recently, several compounds have been discovered that increase the cation permeability of natural and artificial membranes. Among these are a number of amphiphilic macrocyclic molecules that are believed to act as mobile carriers. The antibiotic valinomycin, one of the best studied ionophores, is capable of binding cations, e.g. $\mathrm{K}^{+}$, in apolar media [1]. Evidence that valinomycin acts as a mobile carrier comes from experiments which demonstrate that valinomycin is capable of extracting [2-4] and transporting [3, 5, 6] alkali ions from an aqueous phase into or through an organic phase in the presence of lipophilic anions. 
Kinetic studies with valinomycin to date have been performed only with the black film as a model membrane system [7-15]. It has been shown that in the case of the valinomycin-mediated alkali ion transport through black lipid membranes, the complex formation only occurs at the membrane-water interface [10]. The contribution to the transport of valinomycin-alkali ion complexes, formed in the aqueous phase, proved to be negligible [10]. Moreover, in aqueous media, complex formation only can be detected by very sensitive techniques, such as those which employ fluorescent probes [16].

One of the drawbacks of the black lipid membrane system, especially in studying the effect of variation in the fatty acid composition on the valinomycin-mediated cation transport, is the presence of considerable amounts of solvent in the bilayer structure [17]. Another disadvantage is that such experiments require extended time periods, leading to the uncertainty of adsorption of valinomycin at the walls of the test cells, as discussed in the accompanying paper [18].

Using the liposomal system it has been shown that the valinomycin-mediated exchange diffusion of $\mathrm{Rb}^{+}$against $\mathrm{K}^{+}$is strongly influenced by the fatty acid composition of the constituting phospholipids [19]. At least two possibilities exist that may be responsible for this phenomenon. First, the affinity of the ionophore for the lipid bilayer may increase with increasing unsaturation of the lipid constituents. Secondly, the transport process itself may be dependent on the composition of the membrane lipids.

To extend our knowledge of the valinomycin-induced $\mathrm{K}^{+}$leak, kinetic studies were performed with liposomes containing KCNS. As shown in the accompanying paper [18] the choice of the lipophilic thiocyanate anion as counterion allows the induction of a strong increase in the potassium leak from these liposomes, independent of the presence of uncouplers. The desirability to omit uncouplers is very important because first the complexity of the system is simplified and secondly uncouplers in relatively high concentrations may induce membrane perturbations $[20,21]$ and promote a significant release of $\mathrm{K}^{+}[22]$.

\section{MATERIALS AND METHODS}

Egg lecithin was purified from egg yolk by acetone precipitation and subsequent chromatography over alumina oxide and silica gel. Phosphatidic acid was prepared from egg lecithin by degradation with phospholipase D extracted from Savoy cabbage [23]. Essential phospholipid, lecithin isolated from soybean, was generously supplied by Dr H. Eikermann of Nattermann and Cie, Köln, Germany. Valinomycin was obtained from Calbiochem, Los Angeles, California, U.S.A. All other reagents were commercial and of analytical reagent grade.

Multilayered liposomes were prepared from mixtures of $98 \mathrm{~mole} \%$ lecithin and 2 mole $\%$ phosphatidic acid, as described previously [24]. The outside $\mathrm{K}^{+}$was removed by dialysis against ice-cold, isotonic $150 \mathrm{mM} \mathrm{MgSO}_{4}$. Changes in the $\mathrm{K}^{+}$activity were measured with a potassium-glass electrode (Philips, type G15K), connected with an Ion Activity Meter (Philips, type PW9413) and a recorder.

Measurements were performed at $30^{\circ} \mathrm{C}$, in $150 \mathrm{mM} \mathrm{MgSO}_{4}, 10 \mathrm{mM}$ Tris/ $\mathrm{H}_{2} \mathrm{SO}_{4}$ solutions ( $\mathrm{pH} 7.3$ ), $5.0 \mathrm{ml}$ being the final volume. Valinomycin, dissolved in alcohol, was added with a plumper, $10 \mu \mathrm{l}$ being the maximal amount per $5.0 \mathrm{ml}$. The 
solution was stirred vigorously throughout the course of the experiment. The initial increase of the $\mathrm{K}^{+}$leak was determined by drawing the tangent on the recorder trace at the moment valinomycin was added and correcting for the spontaneous release of $\mathrm{K}^{+}$. The increase of the leak was maximal immediately upon the addition of valinomycin.

Calibration of the electrodes was made in each experiment by adding, in a separate sample under identical experimental conditions, a series of fixed amounts of $\mathrm{K}^{+}$.

\section{RESULTS}

In the specific liposomal system under investigation, only the transport of $\mathrm{K}^{+}$ through the outer bilayer of the multilamellar liposomes contributes directly to the observed leak. If valinomycin is added to the liposomes the increase of the leak is maximal immediately following the addition of the ionophore (Fig. 1). This means that valinomycin has a very high affinity for the lipid bilayer. Furthermore, it strongly suggests that a partition equilibrium of valinomycin between the aqueous phase and the membrane is rapidly established.

Fig. 2 shows that the initial increase of the leak following the addition of valinomycin (from now described as $\Delta \mathrm{K}^{+}$leak) is directly proportional to the valinomycin concentration. Therefore, the amount of valinomycin that actually contributes to the observed $\Delta \mathrm{K}^{+}$leak is directly proportional to the total valinomycin concentration. In order to determine the relationship between valinomycin binding and the presence of $\mathrm{KCNS}$ in the liposomes, the $\Delta \mathrm{K}^{+}$leak was measured from mixtures of liposomes containing $\mathrm{KCNS}$ and liposomes containing $\mathrm{MgSO}_{4}$, at constant total
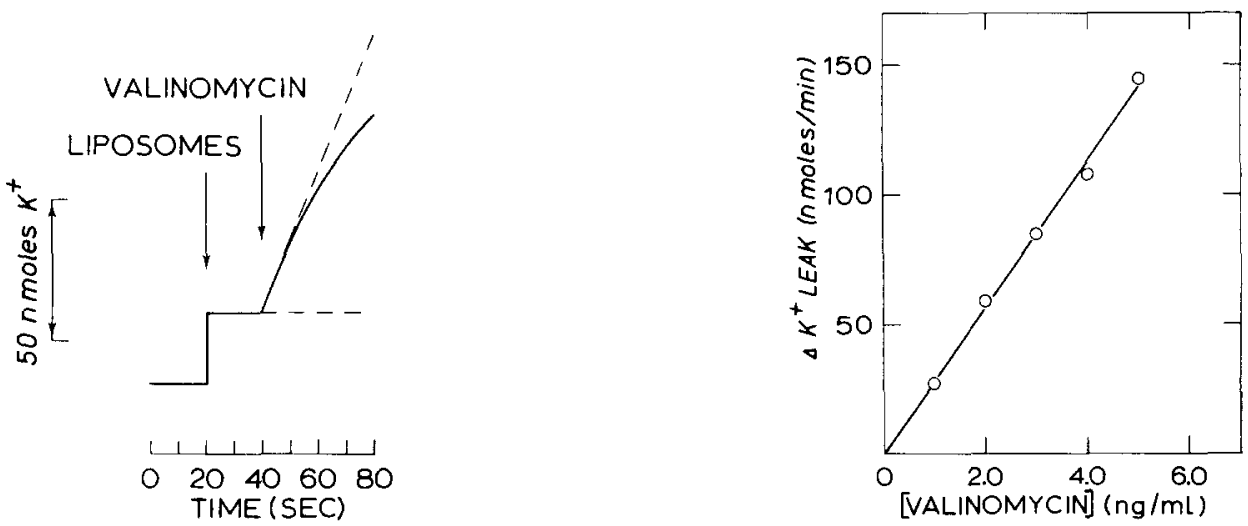

Fig. 1. Recorder trace of the valinomycin-induced leak of $\mathrm{K}^{+}$from egg phosphatidylcholine liposomes. Egg phosphatidylcholine liposomes containing 2 mole \% egg phosphatidic acid were prepared in $150 \mathrm{mM} \mathrm{KCNS}$, followed by dialysis against isotonic $\mathrm{MgSO}_{4}$ solutions at $0-4{ }^{\circ} \mathrm{C}$. The $\mathrm{K}^{+}$leak was measured as described in Materials and Methods in $5.0 \mathrm{ml} 150 \mathrm{mM} \mathrm{MgSO} 4,10 \mathrm{mM}$ Tris $/ \mathrm{H}_{2} \mathrm{SO}_{4}$ (pH 7.3) at $30^{\circ} \mathrm{C}$. Liposome concentration, $0.2 \mu \mathrm{mole} / \mathrm{ml}$; valinomycin concentration, $4.0 \mathrm{ng} / \mathrm{m}$.

Fig. 2. The dependence of the initial increase of the $K^{+}$leak of the valinomycin concentration. Egg phosphatidylcholine liposomes with KCNS enclosed were prepared as in Fig. 1. For experimental details see Materials and Methods. Liposome concentration, $0.2 / \mu \mathrm{mole} / \mathrm{ml}$. Tenperature, $30 \mathrm{C}$. 
liposome concentration (Fig. 3). The results indicate that the $\Delta \mathrm{K}^{+}$leak is directly proportional to the fraction of liposomes containing KCNS; it can then be concluded that the binding of valinomycin is independent of the presence of KCNS in the liposomes.

Fig. 4 demonstrates a non-linear relationship between the $\Delta \mathrm{K}^{+}$leak and the liposome concentration, while the curves in Fig. 5 , in which $1 / \Delta \mathrm{K}^{+}$leak is plotted
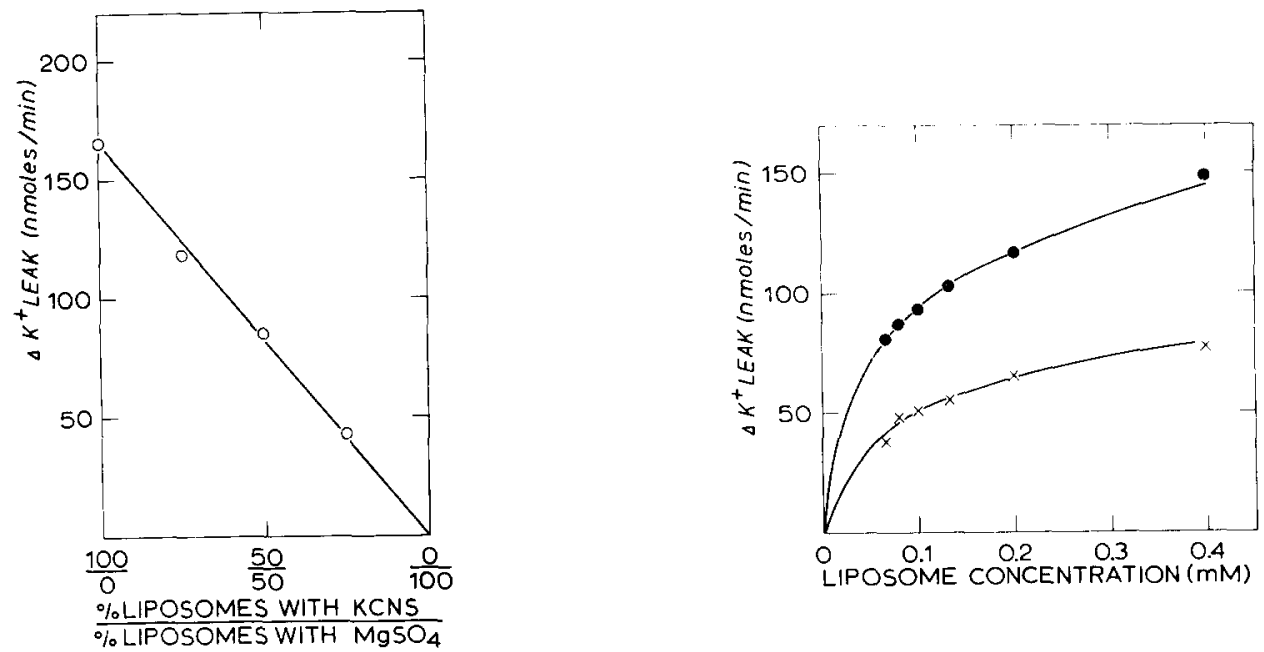

Fig. 3. Measurement of the $\Delta \mathrm{K}^{+}$leak from mixtures of liposomes containing $\mathrm{KCNS}$ or $\mathrm{MgSO}_{4}$ at constant total liposome concentration. Egg phosphatidylcholine liposomes with 2 mole \% egg phosphatidic acid were prepared in $150 \mathrm{mM} \mathrm{KCNS}$ and in $150 \mathrm{mM} \mathrm{MgSO}$ as described in Materials and Methods. Liposome concentration, $0.4 \mu \mathrm{mole} / \mathrm{ml}$; valinomycin concentration, $4.0 \mathrm{ng} / \mathrm{ml}$; temperature $30^{\circ} \mathrm{C}$.

Fig. 4. Measurement of the $\Delta \mathrm{K}^{+}$leak from egg phosphatidylcholine liposomes with $\mathrm{KCNS}$ enclosed at varying liposome concentration. For liposome preparation and measurement of the leak see legend to Fig. 1 and Materials and Methods. Temperature, $30^{\circ} \mathrm{C}$; valinomycin concentration, (...) $2 \mathrm{ng} / \mathrm{ml}$; $(-), 4 \mathrm{ng} / \mathrm{ml}$.

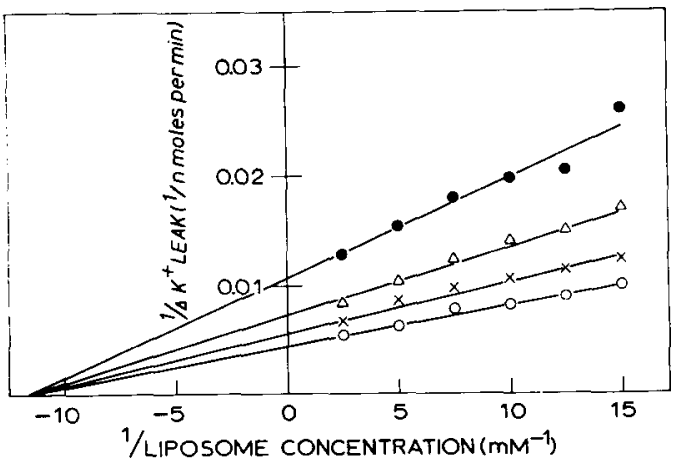

Fig. 5. Double reciprocal plots of the $\Delta \mathrm{K}^{+}$leak at varying liposome concentration. For experimental details see legend to Fig. 4 . Temperature, $30{ }^{\circ} \mathrm{C}$; valinomycin concentration, $(\bigcirc-\bigcirc) 2 \mathrm{ng} / \mathrm{ml} ;(\triangle-\triangle)$ $3 \mathrm{ng} / \mathrm{ml} ;(-\cdots) 4 \mathrm{ng} / \mathrm{ml} ;(\bigcirc-\bigcirc) 5 \mathrm{ng} / \mathrm{ml}$. 


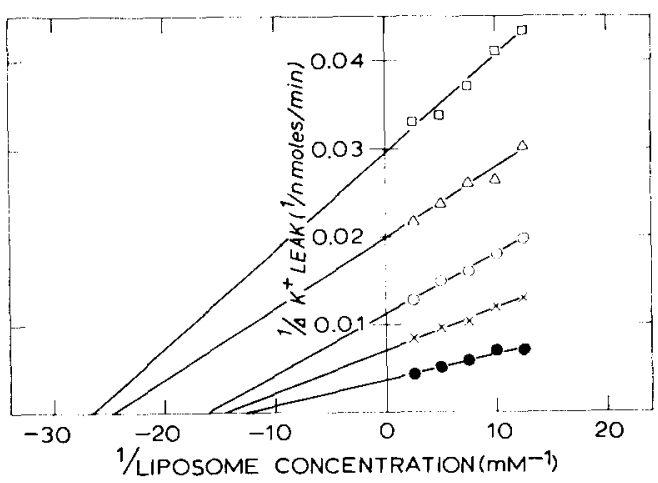

Fig. 6. Temperature dependence of the $A \mathrm{~K}^{+}$leak from egg phosphatidylcholine liposomes with KCNS enclosed. For experimental details see legend to Fig. 4. Valinomycin concentration, $4 \mathrm{ng} / \mathrm{ml}$;

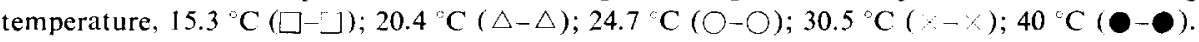
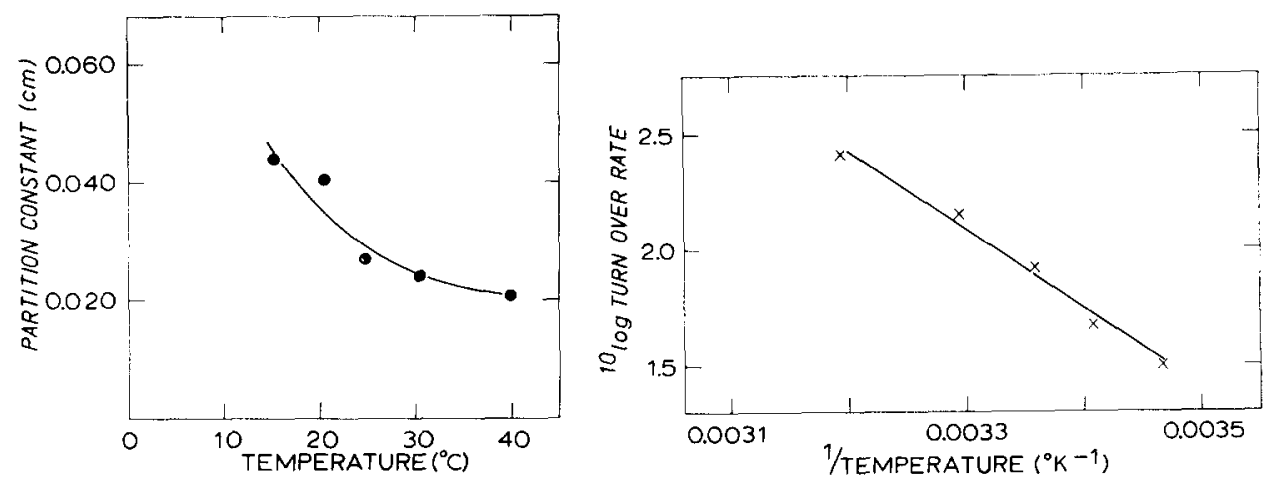

Fig. 7. Temperature dependence of the partition constant. The partition constant was calculated from the curves in Fig. 6 as described in Appendix.

Fig. 8. Arrhenius plot of the turnover rate (ions per molecule valinomycin per min) of the valinomycin induced leak from egg phosphatidylcholine liposomes containing KCNS. The turnover rate was calculated from the curves in Fig. 6 as described in Appendix.

against 1 /liposome concentration, support a hyperbolic dependence of the $\Delta \mathrm{K}^{+}$leak on the liposome concentration. This is in agreement with the assumption that a partition equilibrium exists for valinomycin between the aqueous phase and the liposomal membrane. It follows that for a particular liposome preparation the turnover rate of valinomycin (number of $\mathrm{K}^{+}$transported/molecule valinomycin per $\mathrm{s}$ ) and the constant for the partition equilibrium can be calculated from these curves by extrapolation to $1 /$ liposome concentration $=0$ and $1 / \Delta \mathrm{K}^{+}$leak $=0$, respectively (see Appendix).

The dependence of the $\Delta \mathrm{K}^{+}$leak on the liposome concentration was measured at various temperatures and the results given in Fig. 6 . A plot of the partition constants, calculated from these curves, against temperature shows that the affinity of the antibiotic for the lipid bilayer increases with decreasing temperatures (Fig. 7). Such a negative temperature coefficient for the partition equilibrium of valinomycin was previously reported from studies on black films [25]. Our observation of a negative 

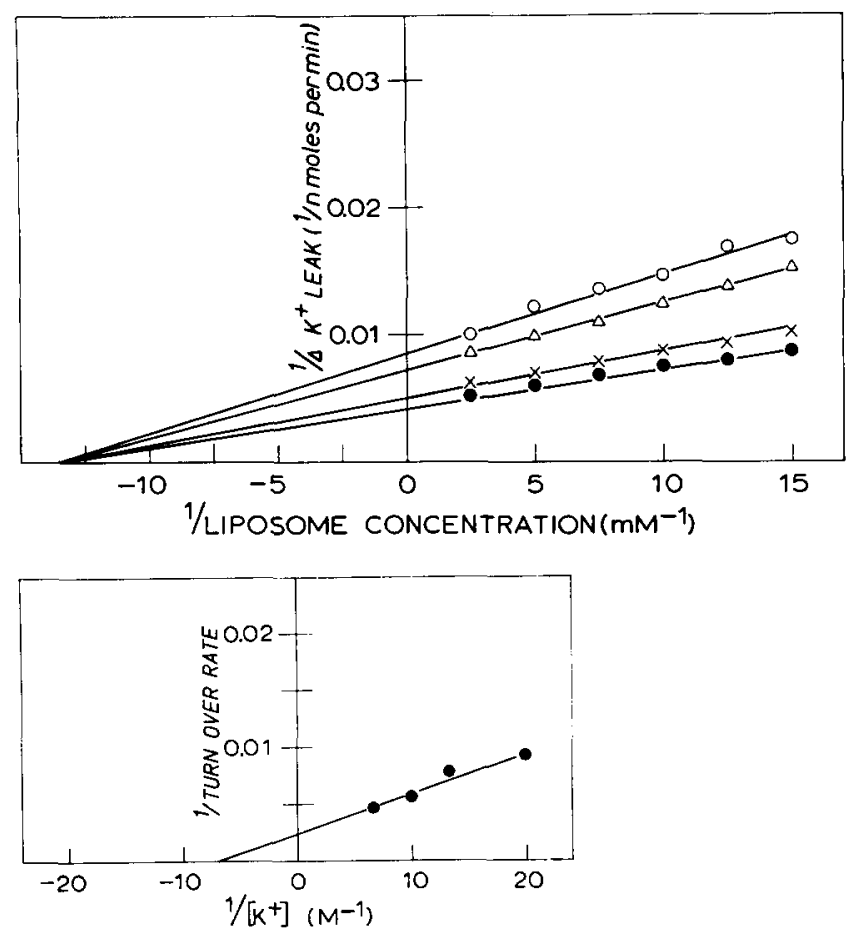

Fig. 9. The dependence of the $\Delta \mathrm{K}^{+}$leak on the $\mathrm{K}^{+}$concentration inside the liposomes. Egg phosphatidylcholine liposomes in KCNS/NaCNS mixtures of varying composition as described in Materials and Methods. Fig. 9a shows the dependence of the $\Delta \mathrm{K}^{+}$leak on the liposome concentration at varying $\mathrm{K}^{+}$concentration inside the liposomes; [KCNS]/[NaCNS] $=150 / 0(\Theta-O) ; 100 / 50(x-)$; $75 / 75(\triangle-\triangle) ; 50 / 100(\bigcirc-\bigcirc) ;(\mathrm{mM} / \mathrm{mM})$. Valinomycin concentration, $4 \mathrm{ng} / \mathrm{ml}$; temperature $30{ }^{\circ} \mathrm{C}$. From these curves the turnover rate (number of $\mathrm{K}^{+} /$molecule valinomycin per $\mathrm{s}$ ) was calculated as described in Appendix. Fig. $9 \mathrm{~b}$ shows the relationship between the turnover rate and the $\mathrm{K}^{+}$concentration inside the liposomes.

temperature coefficient in the present liposomal study provides additional evidence for a rapid establishment of the partition equilibrium. If, in fact, the distribution of the valinomycin between the water and the membrane phase was determining the measured rate of the $\mathrm{K}^{+}$leak, a positive temperature coefficient for the partition equilibrium should be expected. From the Arrhenius plot of the turnover rate against the absolute temperature (Fig. 8) an activation energy for the transport process of $15.7 \pm 1.1 \mathrm{kcals} \cdot \mathrm{mole}^{-1}$ can be calculated. This value is in excellent agreement with $\Delta \mathrm{H}^{*}=15.4 \pm 1.1 \mathrm{kcals} \cdot$ mole $^{-1}$ published by Johnson and Bangham [26] for the valinomycin-induced self diffusion of $\mathrm{K}^{+}$across the liposomal bilayer, measured with radioactive ${ }^{42} \mathrm{~K}^{+}$.

In order to study the transport process in more detail, experiments were done in which the $\mathrm{K}^{+}$and the thiocyanate concentrations inside the liposomes were varied. The $\mathrm{K}^{+}$concentration inside the liposomes was varied by preparing them in $\mathrm{KCNS}$ / $\mathrm{NaCNS}$ mixtures. The influence of the $\mathrm{Na}^{+}$on the potassium transport can be neglected because the affinity of valinomycin for the $\mathrm{Na}^{+}$is at least a factor of $100-1000$ smaller than for the $\mathrm{K}^{+}[3,4,7,13]$. Fig. 9a shows the dependence of the $\Delta \mathrm{K}^{+}$leak 


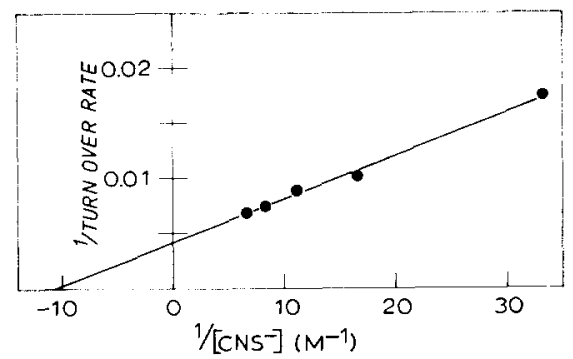

Fig. 10. The dependence of the turnover rate on the thiocyanate concentration inside the liposomes. Liposomes were prepared in $\mathrm{KCNS} / \mathrm{KCl}$ mixtures of varying composition as described in Materials and Methods. The turnover rate was calculated from curves (not shown) as in Fig. 9a as described in Appendix.

on the liposome concentration for four liposome preparations with different $\mathrm{K}^{+}$ concentrations inside. It can be seen that the affinity of the ionophore for the liposomes is not dependent on the $\mathrm{K}^{+}$concentration inside the liposomes. Similar results were found in experiments in which the thiocyanate concentration alone and both the $\mathrm{K}^{+}$and thiocyanate concentrations together inside the liposomes were varied. These findings are in agreement with those of Fig. 3. It can therefore be concluded that, with respect to the partition equilibrium, it is not important whether the ionophore in the membrane is present in the complexed state. In Fig. $9 \mathrm{~b}$ the reciprocal of the turnover rate, calculated from the curves in Fig. 9a, is plotted against the reciprocal of the $\mathrm{K}^{+}$ concentration. The turnover rate appears to be hyperbolically dependent on the $\mathrm{K}^{+}$ concentration in the liposomes. Furthermore, the complex formation cannot be the rate-limiting step of the transport cycle, for, if so, a direct proportionality should appear. In order to study the effect of variation in the thiocyanate concentration on the $\Delta \mathrm{K}^{+}$leak liposomes were prepared in $\mathrm{KCNS} / \mathrm{KCl}$ mixtures. The effect of the presence of $\mathrm{Cl}^{-}$will be negligible because, as is shown in the accompanying paper, the nonlipophilic $\mathrm{Cl}^{-}$permits only a very limited valinomycin-induced $\mathrm{K}^{+}$leak [18]. In Fig. 10 the reciprocal of the turnover rate is plotted against the reciprocal of the thiocyanate concentration. From this result it appears that the turnover rate is also hyperbolically dependent on the thiocyanate concentration inside the liposomes.

To gain further insight into the mechanism of the valinomycin-mediated $\mathrm{K}^{+}$ transport, the effect of a simultaneous variation of the $\mathrm{K}^{+}$and the thiocyanate concentrations on the turnover rate was studied. This was performed in two ways. First, liposomes were made in $\mathrm{KCNS} / \mathrm{NaCl}$ mixtures of varying composition, and secondly liposomes were prepared in KCNS solutions of different molarity followed by dialysis and measurement of the leak against isotonic $\mathrm{MgSO}_{4}$ solutions. Both experiment; gave essentially the same results (Table I). As shown in Fig. 11, a straight line is obtained by plotting the reciprocal of the turnover rate against $1 /\left[\mathrm{K}^{+}\right]\left[\mathrm{CNS}^{-}\right]$.

While studying the effect of variation of the fatty acid composition of the liposomal membrane on the valinomycin-mediated exchange diffusion of $\mathrm{Rb}^{+}$against $\mathrm{K}^{+}$, it was observed that the exchange diffusion increased with increasing unsaturation of the lecithins [19]. The question whether this was due to an increased affinity of the ionophore for the membrane or to an enhancement of the transport process itself could not be answered. The present kinetic studies, however, offer such an 
TABLE I

SOME KINETIC PARAMETERS OF THE VALINOMYCIN-INDUCED $\mathrm{K}^{+}$LEAK FROM LIPOSOMES CONTAINING KCNS AT $30^{\circ} \mathrm{C}$

\begin{tabular}{|c|c|c|c|c|c|}
\hline \multirow[t]{2}{*}{ Experiment } & \multirow[t]{2}{*}{$\begin{array}{l}\text { Liposomes } \\
\text { prepared in: }\end{array}$} & \multirow[t]{2}{*}{$\begin{array}{l}\text { Partition } \\
\text { constant }(\mathrm{cm})\end{array}$} & \multicolumn{2}{|c|}{$\begin{array}{l}\text { Turn-over rate calculated } \\
\text { by extrapolation to: }\end{array}$} & \multirow[t]{2}{*}{ Turn-over rate* } \\
\hline & & & $\mathrm{mM} \mathrm{K}^{+}$ & $\overline{\mathrm{mM} \mathrm{CNS}^{-}}$ & \\
\hline 1 (Fig. 9) & KCNS/NaCNS & 0.0224 & infinite & 150 & $450 \pm 134$ \\
\hline 2 (Fig. 10) & $\mathrm{KCNS} / \mathrm{KCl}$ & 0.0184 & 150 & infinite & $246 \pm 23 \star \star$ \\
\hline 3 (Fig. 11) & $\mathrm{KCNS} / \mathrm{NaCl}$ & 0.0242 & infinite & infinite & $328 \pm 47 \star \star$ \\
\hline \multirow[t]{2}{*}{4} & $\mathrm{KCNS}^{\star \star \star}$ & 0.0239 & infinite & infinite & $288 \pm 33 \star \star$ \\
\hline & & $\overline{0.0222}$ & & & \\
\hline
\end{tabular}

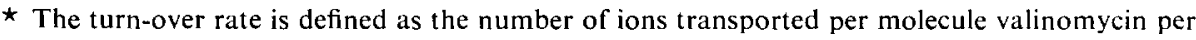
$\mathrm{s}$, calculated by extrapolation as indicated in the Table.

$\star \star$ In these experiments the turn-over rate is equal to the translocation constant (see Appendix).

$\star \star \star$ Liposomes were prepared in KCNS solutions of different molarity, followed by dialysis and measurement of the $A \mathrm{~K}^{+}$leak against isotonic $\mathrm{MgSO}_{4}$ solutions.

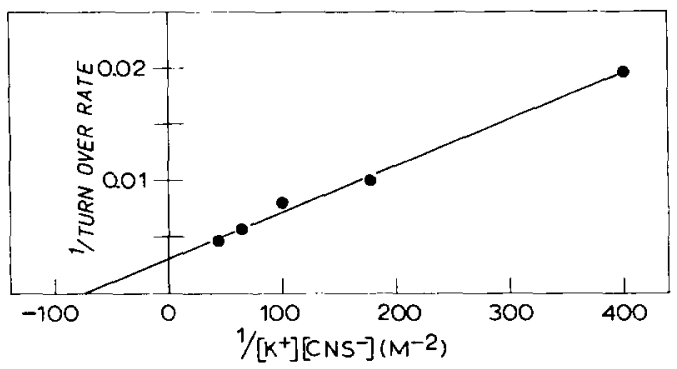

Fig. 11. The dependence of the turnover rate on the KCNS concentration inside the liposomes. Liposomes were prepared in $\mathrm{KCNS} / \mathrm{NaCl}$ mixtures of varying composition as described in Materials and Methods. The turnover rate was calculated from curves (not shown) as in Fig. 9a as described in Appendix.

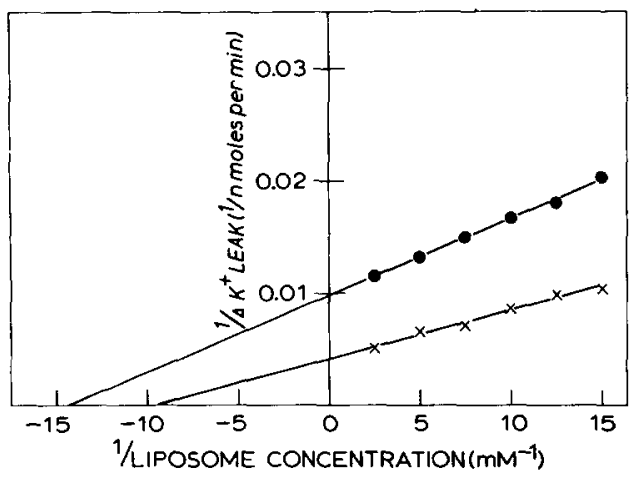

Fig. 12. Double reciprocal plots of the $\Delta \mathrm{K}^{+}$leak at varying liposome concentration from egg lecithin (-) and essential phospholipid $(x-x)$ liposomes. Egg lecithin and essential phospholipids liposomes with 2 mole $\%$ egg phosphatidic acid were prepared in $150 \mathrm{mM}$ KCNS. For experimental details see Fig. 4 . Valinomycin concentration, $2 \mathrm{ng} / \mathrm{ml}$ temperature $30^{\circ} \mathrm{C}$. 
TABLE II

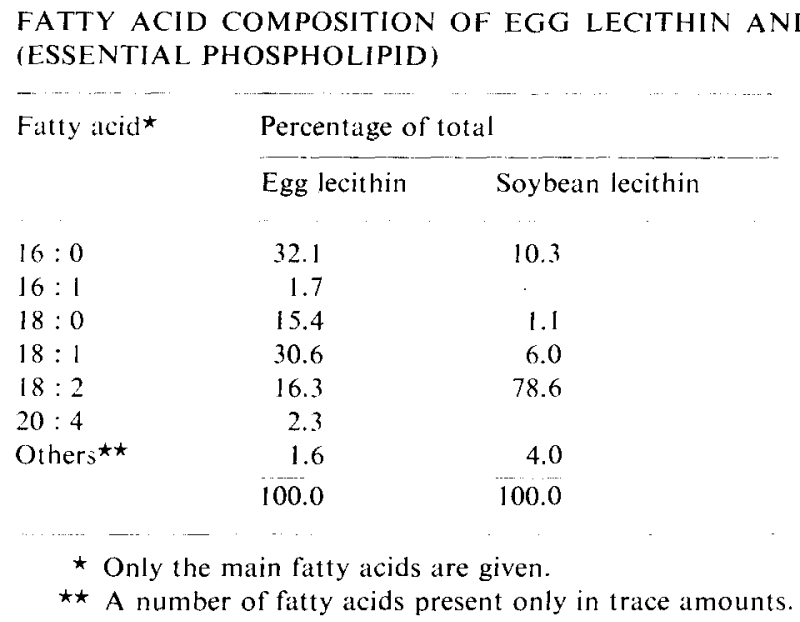

opportunity. We therefore, examined the $\Delta \mathrm{K}^{+}$leak from egg phosphatidylcholine and essential phospholipid liposomes. Essential phospholipid, lecithin isolated from soybean, is in comparison with egg phosphatidylcholine, a highly unsaturated phospholipid, linoleic acid being the predominant fatty acid (Table II). Fig. 12 shows the result of a typical experiment. It is apparent that the turnover rate of valinomycin for essential phospholipid liposomes and egg phosphatidylcholine liposomes, both prepared in $150 \mathrm{mM} \mathrm{KCNS}$, differed significantly, being about 400 and $180 \mathrm{~K}^{+} / \mathrm{mole}-$ cule valinomycin per s respectively. In conclusion then, the valinomycin-mediated transport increases with increasing unsaturation of the membrane constituents, whereas, as can also be seen from Fig. 12, the affinity of the ionophore decreases slightly with increasing unsaturation.

\section{DISCUSSION}

The data collected by measuring the initial increase of the $\mathrm{K}^{+}$leak from liposomes with KCNS enclosed, following the addition of valinomycin, are consistent with the model depicted in Fig. 13.

Concerning the partition of valinomycin between the aqueous phase and the membrane, several lines of evidence indicate that there is a rapid establishment of the partition equilibrium. First, the increase of the leak is maximal immediately upon the addition of the ionophore (Fig. 1). Secondly, the values for the partition constant (Table I, Fig. 7) show that valinomycin has a very high affinity for the lipid bilayer. Thirdly, a negative temperature coefficient for the distribution of valinomycin (Fig. 7 , see also ref. 25) would be unlikely in the absence of a rapidly established equilibrium situation.

A critical question is whether valinomycin is distributed instantaneously over all the bilayers of the multilamellar liposomes or only in the outer bilayer. The high affinity of the ionophore for the lipid bilayer led to the approximation that, during the time course of our experiments, valinomycin was present only in the outer liposomal 


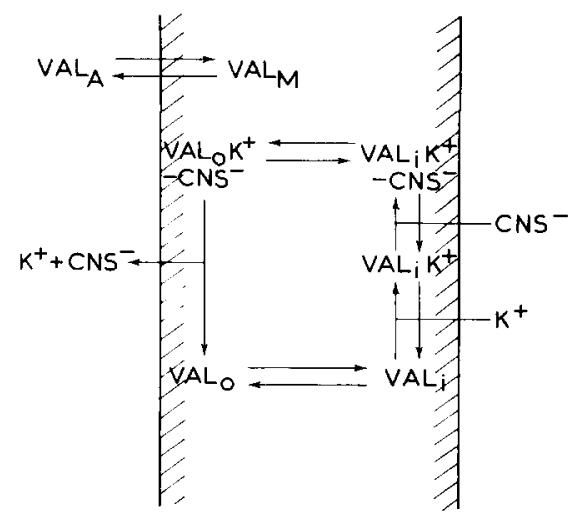

OUTSIDE MEMBRANE INSIDE

Fig. 13. Model for the valinomycin-mediated $\mathrm{K}^{+}$leak from liposomes containing KCNS.

bilayer. In this respect it is relevant that black-film experiments indicate a very slow desorption of valinomycin from the membrane [10,25]. Although a direct comparison may not have full relevance, the value for the partition constant obtained in our experiments $( \pm 0.022 \mathrm{~cm})$ agrees rather well with that from black film studies, ranging from $0.006-0.036 \mathrm{~cm}[10,11,13,15]$. Based on these criteria, we assume that in our experiments in which we measured the initial increase of the $\mathrm{K}^{+}$leak, valinomycin is present only in the outer liposomal bilayer. Additional evidence demonstrated that the partition constant is independent of changes in the $\mathrm{K}^{+}$and the thiocyanate concentrations in the liposomes.

From a comparison of the energetic levels of valinomycin in cyclohexane and toluene-butanol it was concluded that valinomycin is most stable in a semipolar environment and hence will tend to concentrate at membrane-water interfaces [3]. Moreover, it has been shown that valinomycin actively penetrates lecithin monolayers at an air-water interface [1]. So it is likely that, if the diffusion of the uncomplexed carrier is not the rate-limiting step of the transport cycle, the free ionophore will be distributed equally over both interfaces of the bilayer. By changing the $\mathrm{K}^{+}$ concentration inside the liposomes it was found that the turnover rate is hyperbolically dependent on the $\mathrm{K}^{+}$concentration (Fig. 9). This indicates that the complex formation is not the rate-limiting step of the transport cycle. By changing the thiocyanate concentration inside the liposomes and studying the $\Delta \mathrm{K}^{+}$leak (Fig. 10) it appeared that the turnover rate was also hyperbolically dependent on the thiocyanate concentration. These data are in agreement with a model in which it is assumed that a ternary complex is formed of valinomycin with a $\mathrm{K}^{+}$and a thiocyanate ion. If indeed ternary complexes are formed it is plausible that first $\mathrm{K}^{+}$is bound by the ionophore followed by the binding of a thiocyanate ion. Although the formation of ternary complexes has been suggested by several authors $[3,5,6]$ it might be that the electrical coupling of the anion transport to the valinomycin-mediated $\mathrm{K}^{+}$transport kinetically behaves as if ternary complexes are formed. Since the turnover rate at infinite $K^{+}$ concentration and $150 \mathrm{mM} \mathrm{CNS}^{-}$does not differ significantly from that at infinite thiocyanate concentration and $150 \mathrm{mM} \mathrm{K}^{+}$(Table I) this result suggests that the thiocyanate ion has a very high affinity for a valinomycin- $\mathrm{K}^{+}$complex, leaving open 
the question whether a chemical ternary complex is formed or the electrical coupling behaves as if a ternary complex is formed. As discussed in the Appendix, a plot of $1 /$ turnover rate against $1 /\left[\mathrm{K}^{+}\right]\left[\mathrm{CNS}^{-}\right]$will yield a straight line only if the dissociation constant of the ternary complex is much smaller than the thiocyanate concentration inside the liposomes. Fig. 11 shows that within the concentration range of our experiments this assumption holds. From Eqn 15 of the Appendix it can be derived that from the intercept on the X-axis of Fig. 11 the product of the dissociation constants of the equilibria $\mathrm{Val}_{\mathbf{i}}+\mathrm{K}^{+} \stackrel{\mathrm{K}_{1}}{\rightleftharpoons} \mathrm{Val}_{\mathbf{i}} \mathrm{K}^{+}$and $\mathrm{Val}_{\mathbf{i}} \mathrm{K}^{+}+\mathrm{CNS}^{-\stackrel{K_{2}}{\rightleftharpoons}} \mathrm{Val}_{\mathbf{i}} \cdot \mathrm{K}^{+}-\mathrm{CNS}^{-}$can be calculated. Knowing this value the dissociation constant $K_{2}$ can be calculated from the intercept on the $\mathrm{X}$-axis in the graph of the experiment in which the thiocyanate concentration was varied while keeping constant the potassium concentration (Fig. 10). From our experimental data we obtain for $K_{2}$, a value of $0.0042-0.0173 \mathrm{M}$ and for $K_{1} 0.33-1.59 \mathrm{M}$. The value obtained for $K_{1}$ is in good agreement with a value of about $0.6-1.0 \mathrm{M}$ found in black film experiments $[11,13,15]$. In our experiments the lowest thiocyanate concentration inside the liposomes was $0.05 \mathrm{M}$, so $K_{2}$ is much smaller than the thiocyanate concentration.

From the result that the turnover rate is a hyperbolic function of the $\mathrm{K}^{+}$ concentration inside the liposomes it can also be concluded that the diffusion of the free carrier is not rate-limiting. Since the $\mathrm{K}^{+}$concentration in the outside medium is extremely low, the complex formation at the outside of the outer liposomal membrane is negligible. If the diffusion of the free carrier would be rate limiting, uncomplexed valinomycin should accumulate at the outside of the membrane, implying that the turnover rate should no longer be dependent of the $-\mathrm{K}^{+}$concentration inside the liposomes. The significant difference in the turnover rate of valinomycin in egg phosphatidylcholine and essential phospholipid liposomes indicates that the translocation of the complex is the rate-limiting step of the transport cycle. For it is not likely that decomplexation, a process taking place at the membrane-water interface, would depend significantly on the fatty acid composition of the membrane. Comparing our data with those from black film studies, it should be realized that for the liposomal system the enclosed anion plays a significant role with respect to the valinomycininduced $\mathrm{K}^{+}$leak, whereas for black film studies, in which $\mathrm{K}^{+}$is present on both sides of the membrane, the anion is not important. In the black film system the rate constants for the translocation of the valinomycin- $\mathrm{K}^{+}$complex and the free carrier were found to be a factor of 2.5-7 lower than those for the complex formation and decomplexation $[11,13,15]$. This indicates that in the black film system the translocation of the complex was similarly rate limiting. Comparison of the turnover constants derived from these two model systems is complicated by the fact that the experiments were carried out with different lipids and at different temperatures. Furthermore, the fluidity of the black lipid membrane may be significantly influenced by the presence of solvent, e.g. decane, in the film [17]. For the black lipid membrane system with membranes of phosphatidylinositol isolated from brain, and a mixture of soybean phospholipids, turnover constants of about $10^{4}[11,13]$ and and $5 \cdot 10^{3}[15]$ ions $/ \mathrm{mol}-$ ecule valinomycin per s were obtained respectively at $25^{\circ} \mathrm{C}$. On the other hand, we find for egg phosphatidylcholine liposomes at $30^{\circ} \mathrm{C}$ a value of about 328 ions $/ \mathrm{mole}$ cule valinomycin per s (Table I). It is unlikely that such a large difference can be completely ascribed to differences in he lipids used and the temperature of measured 
ment. Rather, the translocation of a valinomycin- $\mathrm{K}^{+}$complex may be much faster than that of a valinomycin- $\mathrm{K}^{+}-\mathrm{CNS}^{-}$complex, and this difference could explain the disparity in values of the turnover constants.

Finally, it should be noted that multilamellar liposomes are suitable for kinetic studies if initial velocities can be measured. In the future more detailed studies are planned to define the effects of variation of the bilayer composition and of phase transitions on the valinomycin-mediated $\mathrm{K}^{+}$transport.

\section{APPENDIX}

In this section we derive the equations that describe the valinomycin-mediated $\mathrm{K}^{+}$leak from liposomes containing KCNS according to the model depicted in Fig. 13. It is assumed that valinomycin distributes rapidly between the aqueous phase and the outer liposomal bilayer. Furthermore, it is assumed that, with respect to the transport process itself, we are also dealing with an equilibrium situation. The following symbols are used:

$A_{0}=$ the total amount of valinomycin (expressed as moles).

$A_{1}=$ the a mount of valinomycin in the aqueous phase $\left(\mathrm{Val}_{\mathrm{A}}\right.$ in Fig. 13).

$A_{2}=$ the amount of valinomycin in the membrane $\left(\mathrm{Val}_{\mathrm{M}}\right)$.

$A_{3}$ and $a_{3}=$ the amount and the concentration, respectively, of uncomplexed valinomycin in the bilayer at the outer $\left(\mathrm{Val}_{0}\right)$ and the inner surface $\left(\mathrm{Val}_{\mathrm{i}}\right)$.

$A_{4}$ and $a_{4}=$ the amount and the concentration of valinomycin-K ${ }^{+}$complexes at the inner surface of the bilayer $\left(\mathrm{Val}_{\mathrm{i}}-\mathrm{K}^{+}\right)$.

$A_{5}$ and $a_{5}=$ the amount and the concentration of ternary complexes at the inner surface of the bilayer $\left(\mathrm{Val}_{\mathrm{i}}-\mathrm{K}^{+}-\mathrm{CNS}^{-}\right)$.

The concentration of valinomycin at the membrane-water interfaces can be expressed as moles valinomycin per $\mathrm{cm}^{2}$ membrane surface.

$b=$ the potassium concentration inside the liposomes (expressed as $\mathbf{M}$ ).

$c=$ the thiocyanate concentration inside the liposomes (expressed as $\mathbf{M}$ ).

$W=$ the volume of the reaction medium $\left(\mathrm{cm}^{3}\right)$.

$N=$ moles of lipid.

First we can say that

$A_{0}=A_{1}+A_{2}$

As discussed in the sections Results and Discussion it appears that as far as the partition equilibrium is concerned the absence or presence of $\mathrm{K}^{+}$and $\mathrm{CNS}^{-}$in the liposomes is not important. In order to describe the partition constant in the usual way as moles valinomycin $/ \mathrm{cm}^{2}$ membranes moles valinomycin $/ \mathrm{cm}^{3}$ medium

it is necessary to know the surface occupied by the outer liposomal bilayer. For handshaken, multilamellar liposomes an outer surface of the liposomes of $303 \mathrm{~cm}^{2}$ per $\mu$ mole of phospholipid has been reported [26]. This means that the total surface, inside and outside, occupied by the outermost liposomal membrane is about $600 \mathrm{~cm}^{2}$ per $\mu$ mole of phospholipid. So the partition constant is given by: 
$K_{\mathrm{p}}=\frac{A_{2} / N 600 \cdot 10^{6}}{A_{1} / W}$

Combining Eqns 1 and 2 gives:

$A_{2}=\frac{600 \cdot 10^{6} K_{\mathrm{p}} N A_{0}}{600 \cdot 10^{6} K_{\mathrm{p}} N+W}$

With respect to the transport cycle itself, it is assumed that the translocation of the complex is the rate-limiting step. The formation of the ternary complex takes place by two distinct steps. First a binary complex is formed: $\mathrm{Val}_{\mathrm{i}}-\mathrm{K}^{+} \rightleftharpoons \mathrm{Val}_{\mathrm{i}}-\mathrm{K}^{+}$. The dissociation constant is given by:

$K_{1}=\frac{a_{3} b}{a_{4}}$

Secondly a ternary complex is formed: $\mathrm{Val}_{\mathbf{i}}-\mathrm{K}^{+}+\mathrm{CNS}^{-} \rightleftharpoons \mathrm{Val}_{\mathbf{i}}-\mathrm{K}^{+}-\mathrm{CNS}^{-}$whose dissociation constant is given by:

$K_{2}=\frac{a_{4} c}{a_{5}}$

The total valinomycin concentration is given by

$a_{2}=2 a_{3}+a_{4}+a_{5}$

Substitution of 4 and 5 in Eqn 6 and elimination of $a_{3}$ and $a_{4}$ yields:

$a_{2}=\frac{2 K_{1} K_{2} a_{5}}{b c}+\frac{K_{2} a_{5}}{c}+a_{5}$

or

$a_{5}=\frac{a_{2} b c}{2 K_{1} K_{2}+\overline{K_{2} b+b c}}$

The concentration of ternary complex $\left(a_{5}\right)$ and the total concentration of valinomycin in the membrane $\left(a_{2}\right)$ are related to the amount of ternary complex $\left(A_{5}\right)$ and the total amount of valinomycin in the membrane $\left(A_{2}\right)$ by $a_{5}=A_{5} / O$ and $a_{2}=A_{2} / O, O$ being the surface occupied by the outer liposomal bilayer. Thus the amount of ternary complex is represented by:

$A_{5}=\frac{A_{2} b c}{2 K_{1} K_{2}+K_{2} b+b c}$

By combining Eqns 3 and $9, A_{2}$ can be eliminated:

$A_{5}=\frac{600 \cdot 10^{6} K_{\mathrm{p}} N A_{\mathrm{o}}}{\left(\frac{600 \cdot 10^{6} \overline{K_{\mathrm{p}} N+W}}{2 K_{1}} \overline{K_{2}+K_{2} b+b c}\right)} b c$

The leak $(L)$ is given by:

$L=k A_{5}$ 
$k$ being the translocation constant of the complex.

Elimination of $A_{5}$ from Eqns 10 and 11 gives:

$\frac{1}{L}=\frac{2 K_{1} K_{2}+K_{2} b+b c}{\left(\frac{600 \cdot 10^{6} K_{\mathrm{p}} N A_{\mathrm{o}}}{600 \cdot 10^{6} K_{\mathrm{p}} N+W}\right) b c k}$

For a given liposome preparation the factor $\left(2 K_{1} K_{2}+K_{2} b+b c / b c k\right)$ will be constant. So, by plotting $(1 / L)$ against $(1 / N)$ a straight line is obtained from which $K_{\mathrm{p}}$ can be calculated from the intercept on the $\mathrm{X}$-axis in which $(1 / L)=0$. In that situation

$K_{\mathrm{p}}=\frac{-W}{606 \cdot 10^{6} N_{1 / L=0}}$

On the other hand, at infinite liposome concentration, Eqn 12 reduces to

$\underline{1}_{L_{1 / N=0}}=\frac{2 K_{1} K_{2}+K_{2} b+b c}{A_{\mathrm{o}} b c k}$

If the turnover rate $(t)$ is defined as $t=L_{1 / N=0} / A_{0}$ and $L_{1 / N=0}$ and $A_{0}$ are eliminated from Eqn 12 we obtain:

$\frac{1}{t}=\frac{2 K_{1} K_{2}+K_{2} b+b c}{b c k}$

From this formula it can be seen that a plot of $1 / t$ against $1 / b$ or $1 / c$ should give straight lines, whereas a plot of $1 / t$ against $1 /(b \cdot c)$ will give a straight line only if $K_{2} \ll c$. As shown in Fig. 11 this proves to be the case and implies that in plots of $1 / t$ against $1 / b$ the intercept on the X-axis approximates $1 / k$ if the concentration of $c \gg K_{2}$. An estimation of $K_{2}$, calculated from Figs 10 and 11 , shows that under our experimental conditions $K_{2}$ was about a factor of 10 smaller than $c$, whereas values for $K_{1}$ are obtained which are in good agreement with the value reported from black lipid membrane studies $[11,13,15]$.

\section{ACKNOWLEDGEMENTS}

The present investigations have been carried out under the auspices of the Netherlands Foundation for Biophysics and with financial aid from the Netherlands Organization for the Advancement of Pure Research (ZWO).

\section{REFERENCES}

1 Shemyakin, M. M., Ovchinnikov, Yu. A., Ivanov, V. T., Antonov, V. K., Vinogradova, E. I., Shkrob, A. M., Malenkov, G. G., Evstratov, A. V., Laine, I. A., MeInik, E. I. and Ryabova, I. D. (1969) J. Membr. Biol. 1, $402-430$

2 Pressman, B. C., Harris, E. J., Jagger, W. S. and Johson, J. H. (1967), Proc. Nat. Acad. Sci. U.S. 58, 1949-1956

3 Pressman, B. C. and Haynes, D. H. (1969) in The molecular basis of membrane function (Tosteson, D. C. ed.), pp. 221-246, Englewood Cliffs, N. J., Prentice-Hall

4 Eisenman, G., Szabo, G., McLaughlin, S. G. A. and Ciani, S. M. (1973) Bioenergetics, 4, 93-148 
5 Pinkerton, M., Steinrauf, L. K. and Dawkins, P. (1969) Biochem. Biophys. Res. Comm. 35, $512-518$

6 Ashton, R. and Steinrauf, L. K. (1970) J. Membr. Biol. 49, 547-556

7 Mueller, P. and Rudin, D. O. (1967) Biochem. Biophys. Res. Comm. 26, 398404

8 Liberman, E. A. and Topaly, V. P. (1968) Biochim. Biophys. Acta 163, 125-136

9 Tosteson, D. C. (1968) Fed. Proc. 27, 1269-1277

10 Stark, G. and Benz, R. (1971) J. Membr. Biol. 5, 133-153

11 Stark, G., Ketterer, B., Benz, R. and Laüger, P. (1971) Biophys. J. 11, 981-994

12 Krasne, S., Eisenman, G. and Szabo, G. (1971) Science 174, 412-415

13 Laüger, P. (1972) Science 178, 24-30

14 Stark, G. (1972) Biochim. Biophys. Acta 298, 323-332

15 Gambale, F., Gliozzi, A. and Robello, M. (1973) Biochim. Biophys. Acta 330, 325-334

16 Feinstein, M. B. and Felsenfeld, H. (1971) Proc. Nat. Acad. Sci. U.S.A. 68, 2037-2041

17 Pagano, R. E., Ruysschaert, J. M. and Miller, I. R. (1972) J. Membr. Biol. 10, 11-30

18 Blok, M. C., de Gier, J. and van Deenen, L. L. M. (1974) Biochim. Biophys. Acta 367, 209-202

19 de Gier, J., Haest, C. W. M., Mandersloot, J. G. and van Deenen, L. L. M. (1970) Biochim. Biophys. Acta 211, 373-375

20 Zimmer, G., Keith, A. D. and Packer, L. (1972) Arch. Biochem. Biophys. 152, 105-113

2 I Verma, S. P., Schneider, H. and Smith, I. C. P. (1973) Arch. Biochem. Biophys. 154, 400-406

22 Scarpa, A. and de Gier, J. (1971) Biochim. Biophys. Acta 241, 789-797

23 Davidson, J. M. and Long, C. (1958) Biochem. J. 69, 458-466

24 de Gier, J., Mandersloot, J. G. and van Deenen, L. L. M. (1968) Biochim. Biophys. Acta 150 , $666-675$

25 Stark, G., Benz, R., Pohl, G. W. and Janko. K. (1972) Biochim. Biophys. Acta 266, 603-612

26 Johnson, S. M. and Bangham, A. D. (1969) Biochim. Biophys. Acta 193, 92-104

27 Bangham, A. D., de Gier, J. and Greville, G. D. (1967) Chem. Phys. Lipids 1, 225-246 